Full-length article

\title{
Specific targeting of gliomas with multifunctional superparamagnetic iron oxide nanoparticle optical and magnetic resonance imaging contrast agents $^{1}$
}

\author{
Xiang-xi MENG ${ }^{2}$, Jia-qi WAN ${ }^{4}$, Meng JING ${ }^{2}$, Shi-guang ZHAO ${ }^{2}$, Wei CAI ${ }^{3}$, En-zhong LIU $^{2,4}$ \\ ${ }^{2}$ Department of Neurosurgery, First Affiliated Hospital, Harbin Medical University, Harbin 150001, China; ${ }^{3}$ School of Material Science and \\ Engineering, Harbin Institute of Technology, Harbin 150001, China
}

\section{Key words}

superparamagnetic iron oxide; nanoparticles; gliomas; biocompatibility; magnetic resonance imaging; optical imaging; molecular imaging

\footnotetext{
${ }^{1}$ Project supported by the National Natural Science Foundation of China (№ 30672154 and No 30371458) and the Science and Technology Research Project of the Department of Education of Heilongjiang Province (No 11511191 ).

${ }^{4}$ Correspondence to Prof En-zhong LIU. Phn 86-451-8426-0286.

Fax 86-451-5363-3660.

E-mail xiangximeng@gmail.com

Received 2006-12-04

Accepted 2007-05-16

doi: $10.1111 / \mathrm{j} .1745-7254.2007 .00661 . \mathrm{x}$
}

\begin{abstract}
Aim: To determine whether glioma cells can be specifically and efficiently targeted by superparamagnetic iron oxide nanoparticle (SPIO)-fluorescein isothiocyanate (FITC)-chlorotoxin (SPIOFC) that is detectable by magnetic resonance imaging (MRI) and optical imaging. Methods: SPIOFC was synthesized by conjugating SPIO with FITC and chlorotoxin. Glioma cells (human U251-MG and rat C6) were cultured with SPIOFC and SPIOF (SPIO-FITC), respectively. Neural cells were treated with SPIOFC as the control for SPIOFC-targeted glioma cells. The internalization of SPIOFC by glioma cells was assessed by MRI and was quantified using inductively-coupled plasma emission spectroscopy. The optical imaging ability of SPIOFC was evaluated by confocal laser scanning microscopy. Results: Iron per cell of U251 ( $72.5 \pm 1.8 \mathrm{pg})$ and C6 (74.9 $\pm 2.2 \mathrm{pg})$ cells cultured with SPIOFC were significantly more than those of U251 $(6.6 \pm 1.0 \mathrm{pg})$ and C6 (7.1 \pm 0.8 $\mathrm{pg}$ ) cells incubated with SPIOF. The $\mathrm{T}_{2}$ signal intensity of U251 and $\mathrm{C} 6$ cells cultured with SPIOFC (233.6 25.9 and $211.4 \pm 17.2$, respectively) were substantially lower than those of U251 and C6 cells incubated with SPIOF (2275.3 \pm 268.6 and $2342.7 \pm 222.4$, respectively). Moreover, there were significant differences in iron per cell and $\mathrm{T}_{2}$ signal intensity between SPIOFC-treated neural cells (1.3 \pm 0.3 ; $2533.6 \pm 199.2)$ and SPIOFC-treated glioma cells. SPIOFC internalized by glioma cells exhibited green fluorescence by confocal laser scanning microscopy. Conclusion: SPIOFC is suitable for the specific and efficient targeting of glioma cells. MRI and optical imaging in conjunction with SPIOFC can differentiate glioma cells from normal brain tissue cells.
\end{abstract}

\section{Introduction}

Gliomas are the most common and mortiferous type of primary brain tumors ${ }^{[1,2]}$. The differentiation of central gliomas from peripheral brain tissue is very crucial for accurate resection of all diseased tissues. The effectiveness of tumor resection is severely limited by the poor visual contrast between gliomas and normal brain tissue by gadolinium chelate-enhanced magnetic resonance imaging (MRI) ${ }^{[3]}$. Superparamagnetic iron oxide nanoparticles (SPIO) have also been widely used for MRI contrast enhancement ${ }^{[4-6]}$, and labeled as glioma cells to visualize tumors at real cell-level resolution by MRI ${ }^{[3,7-9]}$. Moreover, fluorophores can be conjugated with SPIO to visualize labeled glioma cells in optical imaging ${ }^{[10]}$. However, the low specificity of intracellular uptake by gliomas limits the extensive use of SPIO. SPIO were also internalized by reactive astrocytes and macrophages ${ }^{[3,10,11]}$, which lead to overestimating the extent of gliomas. Therefore, to achieve specific glioma cell recognition and increased efficiency of the SPIO intracellular uptake, a novel SPIO MRI contrast agent for specific targeting gliomas needs to be developed.

Nanoparticles can be internalized into cells generally via 
receptor-mediated endocytosis ${ }^{[12]}$. One strategy to realize the specific and efficient intracellular uptake of nanoparticles is to modify the nanoparticles surface with a ligand which is preferentially and efficiently taken up by target cells via receptor-mediated endocytosis ${ }^{[13-15]}$. Chlorotoxin is a small 36-amino acid peptide that was originally isolated from the giant Israeli scorpion (Leiurus quinquestriatus) venom ${ }^{[16,17]}$. Chlorotoxin specific receptor on the surface of glioma cells was proven to be membrane-bound matrix metalloproteinase-2 (MMP-2), which binds with high affinity to chlorotoxin ${ }^{[18]}$. MMP-2 is specifically upregulated in gliomas and other tumors of neuroectodermal origin, but is not normally expressed in tumors of non-neuroectodermal origin and normal brain tissue, including neural cells ${ }^{[19]}$. MMP-2 can specifically inhibit the glioma-specific chloride channel which only exists on glioma cells rather than normal brain tissue cells, including neural cells. Therefore, chlorotoxin is capable of binding to gliomas cells with highly specific and selective targeting characters ${ }^{[20,21]}$. Despite the specific targeting role of chlorotoxin nanoparticles conjugated to $9 \mathrm{~L}$ gliosarcoma cells having been confirmed ${ }^{[22]}$, little effort has been made on the basis of glioma cells of human origin. Investigating the specific targeting role of chlorotoxinnanoparticles conjugated to human U251 glioma cells will set a theoretical foundation for following an in vivo study. Moreover, the appropriate biocompatibility of conjugated chlorotoxin nanoparticles was essential for introducing the conjugated material into clinical practice. Therefore, the present study paid additional attention on the biocompatibility of conjugated chlorotoxin nanoparticles.

In this study, SPIO-fluorescein isothiocynate (FITC)chlorotoxin (SPIOFC) was synthesized for improving the specificity of SPIO for targeting glioma cells. The biocompatibility of SPIOFC was examined to estimate the value of its biomedical application. Prussian blue staining and MRI were utilized to investigate the intracellular uptake of SPIOFC by human U251-MG and rat C6 glioma cells in order to reflect the actual specificity of SPIOFC for targeting glioma cells. Inductively-coupled plasma emission spectroscopy (ICP) was employed to quantify the intracellular uptake of SPIOFC. Confocal laser scanning microscopy was employed to evaluate the optical imaging ability of SPIOFC.

\section{Materials and methods}

Newborn Wistar rats (Harbin Medical University Experimental Animals, Harbin, China) were used in this study. The experiments were conducted in accordance with the Guide for the Care and Use of Laboratory Animals as adopted and promulgated by the Declaration of Helsinki.

Reagents 3-Aminopropyl-triethoxy-silane (APS), bicinchoninic acid (BCA) kit, FITC, 3-(4,5-dimethylthiazol-2-yl)-2, 5-diphenyl-tetrazolium bromide (MTT), Nuclear Fast Red solution, and gelatin were purchased from Sigma (St Louis, MO, USA). Chlorotoxin was obtained from AnaSpec (San Jose, CA, USA). Fetal bovine serum (FBS), Dulbecco's modified Eagle's medium (DMEM), Dulbecco's Modified Eagle's Medium: Nutrient Mixture F-12 (DMEM/F-12), and B-27 supplements were purchased from Gibco (Grand Island, New York, USA).

Synthesis of multifunctional specific targeting contrast agent for gliomas (SPIOFC) SPIOFC consisted of SPIO, FITC, and chlorotoxin. Water-soluble SPIO ( $7 \pm 1.6 \mathrm{~nm}$ in size $)$ were prepared via our previously published approach ${ }^{[23,24]}$. Fluorescent and magnetic bifunctional core-shell nanoparticles (SPIOF) were prepared by encapsulating SPIO cores in a FITC dropped silica shell using a sol-gel approach ${ }^{[25]}$. Aminomodified SPIOF were obtained by hydrolyzing APS in SPIOF suspension solution. Then chlorotoxin was covalently conjugated with the surface amine groups on SPIOF to form SPIOFC via a standard covalently binding method as described by Weissleder et $a l^{[26]}$. The morphology of SPIOFC was investigated by transmission electron microscope (TEM) on a Philips Tencai 20 electron microscope (Eindhoven, Netherlands) at an accelerating voltage of $200 \mathrm{kV}$. The amount of chlorotoxin immobilized on the contrast agent was quantified using a BCA kit for the protein assay.

Cells and cell cultures Human U251-MG glioma and rat C6 glioma cell lines were kindly provided by Keiji KAWAMOTO (Department of Neurosurgery, Kansai Medical University, Osaka, Japan), and were routinely cultured at $37^{\circ} \mathrm{C}$ in a humidified atmosphere with $5 \% \mathrm{CO}_{2}$ in DMEM supplemented with $10 \%$ FBS, $100 \mathrm{kU} / \mathrm{L}$ penicillin, and 100 $\mathrm{mg} / \mathrm{L}$ streptomycin. Cell viability and density were determined through staining with trypan blue. Cell counts were obtained using a hemocytometer.

Neural cells were prepared from the cortex of newborn Wistar rats in accordance with the methods described previously ${ }^{[27]}$. In brief, the cortical tissue was carefully freed from blood vessels and meninges. The tissue was trypsinized for $20 \mathrm{~min}$, carefully disintegrated with a fire-polished pipette, and washed twice. The cortical cells were cultured in DMEM/ F-12 medium supplemented with $10 \% \mathrm{FBS}, 100 \mathrm{kU} / \mathrm{L}$ penicillin, $100 \mathrm{mg} / \mathrm{L}$ streptomycin, and 2\% B-27 supplements. The medium was changed every third day.

Cytotoxicity assay by MTT The MTT assay was performed to evaluate whether SPIO, chlorotoxin, and SPIOFC impair the viability of glioma cells and neural cells using corresponding untreated cells as the control. The cells were 
seeded onto 96 -well plates at a density of $6 \times 10^{3}$ cells/well for $24 \mathrm{~h}$ (U251-MG and C6 glioma cells) and $7 \mathrm{~d}$ (neural cells), respectively. Then SPIO at final concentrations of 5, 10, 25, $50,75,100$, and $200 \mathrm{mg} / \mathrm{L}$ (iron concentrations), chlorotoxin at concentrations of $0.8,1.6,2.4,3.2,4.0,4.8$, and $5.6 \mu \mathrm{mol} / \mathrm{L}$, and SPIOFC at final concentrations of $100 \mathrm{mg} / \mathrm{L}$ (iron concentrations) and $1.6 \mu \mathrm{mol} / \mathrm{L}$ (chlorotoxin concentration), respectively, were added to the cells and incubated for $24 \mathrm{~h}$. The cells were washed twice with phosphate-buffered saline (PBS) and replenished with fresh medium, followed by incubation for a further $48 \mathrm{~h}$. Then the cells were examined by MTT assay.

Cytochemistry analysis Prussian blue staining was employed to determine the presence of iron inside the cells. Untreated cells were seeded onto 6-well plates at a density of $3 \times 10^{5}$ cells/well. Twenty four hours after seeding, glioma cells (human U251-MG and rat C6) were cultured with SPIOFC and SPIOF at a concentration of $100 \mathrm{mg} / \mathrm{L}$ for $1 \mathrm{~h}$. Seven days after seeding, neural cells were treated with SPIOFC at a concentration of $100 \mathrm{mg} / \mathrm{L}$ for $1 \mathrm{~h}$ as the control for SPIOFCtargeted glioma cells. Following labeling, the samples were washed twice with cell culture medium and twice with PBS. The cells were fixed with $4 \%$ paraformaldehyde solution and washed with PBS, followed by incubation in Perl's solution (equal parts of $6 \%$ hydrochloric acid and $2 \%$ potassium ferrocyanide) for 30 min. After washing with deionized water, the treated cells were counterstained with Nuclear Fast Red solution. The specimens were then mounted and examined under a light microscope. The percentage of Prussian blue stained-positive cells was counted under a random field of view $(\times 200)$ and the procedure was repeated 20 times for each specimen.

Quantification of nanoparticle intracellular uptake The intracellular iron concentrations were quantified using ICP (Perkin-Elmer Optima 5300 DV, Perkin Elmer, Beaconsfield, UK). After being cultured with the abovementioned nanoparticles, the cells were washed with PBS, detached, resuspended, counted, centrifuged down, and the cell pellet was dissolved in $37 \% \mathrm{HCl}$ solution at $70-80{ }^{\circ} \mathrm{C}$ for 30 min. The samples were diluted to final iron concentrations of 1.0-4.0 mg/L. Three replicates were measured.

MRI For MRI, the specimens were prepared by suspending $2 \times 10^{6}$ cells in $100 \mu \mathrm{L}$ of $4 \%$ gelatin. The cell suspensions were seeded onto 96-well plates at a volume of $100 \mu \mathrm{L} /$ well and allowed to solidify at $4{ }^{\circ} \mathrm{C}$. The spaces surrounding each well were full of deionized water to allow appropriate image acquisition. MRI was performed with a 1.5T clinical MR imager (TOSHIBA Visart, Japan) using a hu-

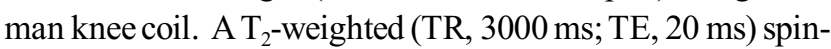
echo sequence was selected to acquire the magnetic resonance (MR) images. The spatial resolution parameters were as follows: an acquisition matrix of $256 \times 192$, field of view of $15 \times 15 \mathrm{~cm}$, section thickness of $2.5 \mathrm{~mm}$, and thickness gap of $0.5 \mathrm{~mm}$. The regions of interest of $0.12 \mathrm{~cm}^{2}$ were placed in the center of each sample image to obtain signal intensity measurements using the provided image quantification tool.

Confocal laser scanning microscopy Confocal laser scanning microscopy was employed to ensure the optical imaging ability of SPIOFC for visualizing gliomas. As mentioned above, $1 \times 10^{5}$ cells (human U251-MG and rat C6 glioma cells) were seeded onto coverslips $24 \mathrm{~h}$ prior to labeling and imaging. Then the cells were cultured with SPIOFC for $1 \mathrm{~h}$. After the cells were labeled by SPIOFC, the coverslips were washed twice with cell culture medium and several times with PBS buffer. The confocal images of the labeled cells were obtained with a Zeiss LSM 510 META confocal laser scanning microscope (Zeiss, Oberkochen, Germany). A 488 $\mathrm{nm} \mathrm{Ar}$ ion laser was used to excite the green fluorescence of FITC (510-525 nm).

Data process and statistical analysis It was difficult to determine the absolute value of cell viability. Therefore, the viability of the untreated cells was considered as $100 \%$ control. Then the viability of cells with SPIO, chlorotoxin, and SPIOFC was expressed as the percentage of absorbance of the control untreated cells. Data were presented as mean \pm SD. ANOVA was used to compare cell viability, and $P<0.05$ indicated significant statistical differences in ANOVA. The Kruskal-Wallis test was used to compare percentages of Prussian blue stained-positive cells, qualification of intracellular iron, and $\mathrm{T}_{2}$ signal intensity; $P<0.0125$ was considered to be statistically significant.

\section{Results}

Characters of SPIOFC The morphology of SPIOFC was analyzed by TEM as shown in Figure 1. The TEM image showed that the core-shell structure SPIOFC was comprised of the core (SPIO) and homogeneous silica shell. The final

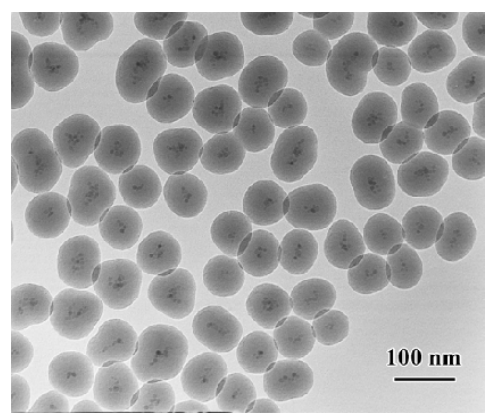

Figure 1. TEM image of SPIOFC. Core-shell structure SPIOFC was comprised of the SPIO core and homogeneous silica shell. 
diameter of SPIOFC was $80 \pm 15.6 \mathrm{~nm}$. The average number of chlorotoxin molecules per SPIOFC was calculated to be approximate 50.2 using a BCA kit for the protein assay.

Cytotoxicity assay by MTT The cell viability of the SPIOtreated cells (human U251-MG and rat C6 glioma cells, and neural cells) was not statistically less than that of the corresponding untreated cells. The viability of U251-MG (Figure 2A) and C6 glioma cells (Figure 2B), and neural cells (Figure 2C) ranged from $92.4 \% \pm 17.3 \%$ to $101.8 \% \pm 11.0 \%, 94.7 \% \pm 23.3 \%$ to $106.8 \% \pm 16.2 \%$, and $90.0 \% \pm 14.5 \%$ to $123.0 \% \pm 20.6 \%$, respectively, whereas the viability of untreated U251-MG and C6 glioma cells, and neural cells was $100.0 \% \pm 7.5 \%$, $100.0 \% \pm 16.5 \%$, and $100.0 \% \pm 14.4 \%$, respectively. This result suggests that SPIO at the abovementioned concentrations have no obvious cytotoxicity on the 3 cell types.

The viability of the untreated U251-MG and C6 glioma cells was $100.0 \% \pm 15.8 \%$ and $100.0 \% \pm 14.7 \%$, respectively. The cell viability of the chlorotoxin $(0.8$ and $1.6 \mu \mathrm{mol} / \mathrm{L})$-treated gliomacells was $101.3 \% \pm 17.7 \%, 98.1 \% \pm 23.7 \%, 98.4 \% \pm 14.4 \%$, and $94.8 \% \pm 19.9 \%$ for human U251-MG (Figure $3 \mathrm{~A}$ ) and rat C6 cells (Figure 3B), respectively. This suggested that chlorotoxin at the abovementioned concentrations did not impair the glioma cell viability. In contrast, the cell viability of the chlorotoxin $(2.4,3.2,4.0,4.8$, and $5.6 \mu \mathrm{mol} / \mathrm{L})$-treated glioma cells ranged from $67.6 \% \pm 13.6 \%$ to $83.8 \% \pm 15.1 \%$ and $67.7 \% \pm 14.2 \%$ to $80.8 \% \pm 9.3 \%$ for human U251-MG (Figure $3 \mathrm{~A}$ ) and rat C6 cells (Figure 3B), respectively. This suggested that chlorotoxin at the specific concentrations impaired glioma cell viability. Moreover, the cell viability of the chlorotoxin-treated neural cells was not statistically less than that of the corresponding untreated cells (Figure 3C).

SPIOFC was then synthesized by SPIO at a concentration of $100 \mathrm{mg} / \mathrm{L}$ (iron concentrations) and chlorotoxin at the concentration of $1.6 \mu \mathrm{mol} / \mathrm{L}$ for the following measurement. The cell viability of the SPIOFC-treated cells (U251-MG and rat C6 glioma cells, and neural cells) was not statistically lower than that of the control untreated cells. The viability of the SPIOFC-treated U251-MG (Figure 4A) and C6 glioma
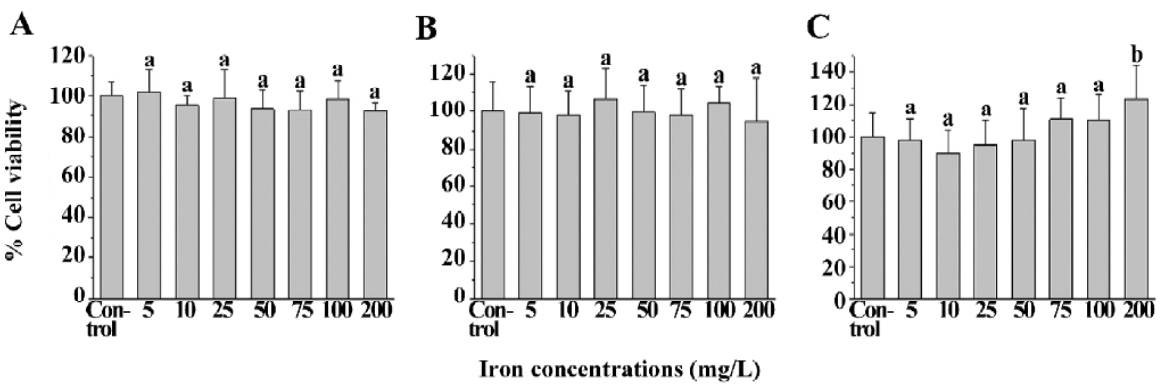

Figure 2. Viability of human U251-MG glioma cells (A), C6 glioma cells (B), and neural cells (C). Cells were cultured with SPIO at concentrations of $5,10,25,50,75,100$, and $200 \mathrm{mg} / \mathrm{L}$ ( $n=11$ for each concentration group), respectively. SPIO at the abovementioned concentrations did not impair cell viability. Cell viability is expressed as the mean $\pm \mathrm{SD}$ of the percentage of absorbance of the controls where $100 \%$ equals the viability of untreated control cells $(n=16)$. ${ }^{\mathrm{a}} P>0.05,{ }^{\mathrm{b}} P<0.05$ vs corresponding control group.

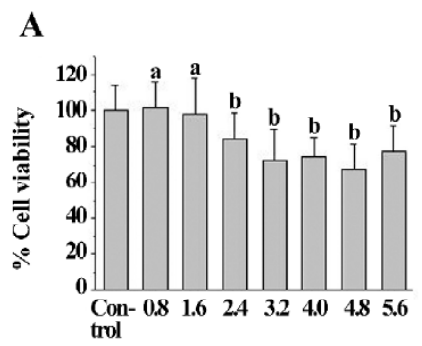

B

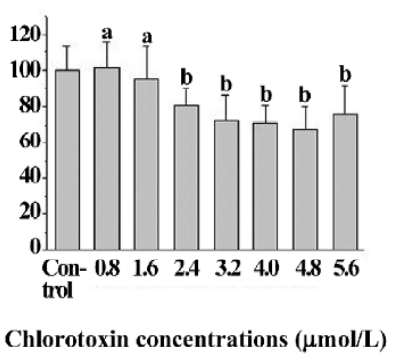

C

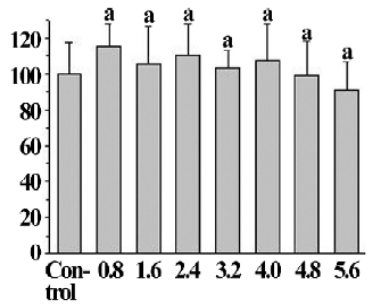

Figure 3. Viability of human U251-MG glioma cells (A), C6 glioma cells (B), and neural cells (C). Cells were cultured with chlorotoxin at concentrations of $0.8,1.6,2.4,3.2,4.0,4.8$, and $5.6 \mu \mathrm{mol} / \mathrm{L}$, respectively. Chlorotoxin at low concentrations $(0.8$ and $1.6 \mu \mathrm{mol} / \mathrm{L})$ did not impair the viability of glioma cells, whereas high concentrations of chlorotoxin $(2.4,3.2,4.0,4.8$, and $5.6 \mu \mathrm{mol} / \mathrm{L}) \mathrm{had}$ cytotoxic effects on glioma cells. Chlorotoxin at all the abovementioned concentrations did not impair the viability of neural cells. $n=11 ;$ mean \pm SD. ${ }^{\mathrm{a}} P>0.05,{ }^{\mathrm{b}} P<0.05 v s$ corresponding control group. 

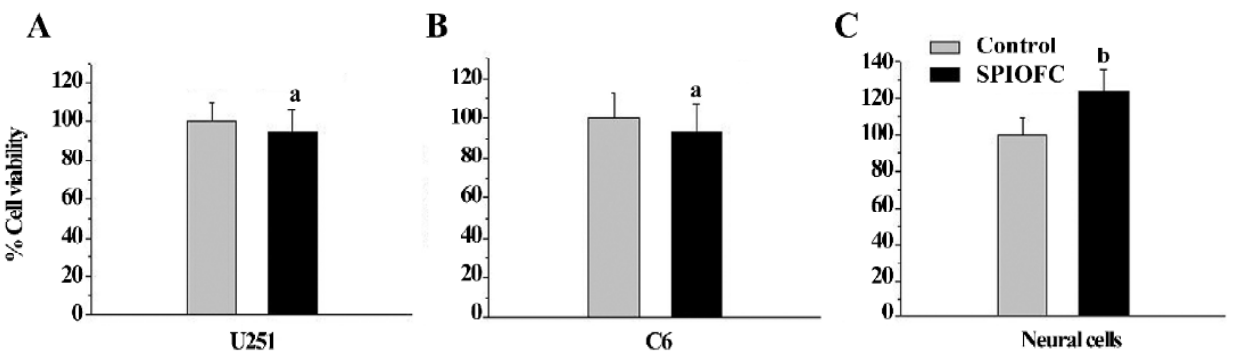

Figure 4. Viability of human U251-MG glioma cells (A), C6 glioma cells (B), and neural cells (C). Cells were cultured with SPIOFC at a concentration of $100 \mathrm{mg} / \mathrm{L}$ ( $n=16$ for each cell line). SPIOFC has no deleterious effects on the aforementioned cells. Mean $\pm \mathrm{SD}$. ${ }^{\mathrm{a}} P>0.05$, ${ }^{\mathrm{b}} P<0.05$ vs corresponding control group ( $n=13$ for U251-MG and neural cells; $n=16$ for C6 cells).

cells(Figure 4B), and neural cells (Figure 4C) was 94.6\% $\pm 11.7 \%$, $93.3 \% \pm 14.3 \%$, and $126.8 \% \pm 11.9 \%$, respectively, and the viability of the untreated U251-MG and C6 glioma cells, and neural cells was $100.0 \% \pm 12.3 \%, 100.0 \% \pm 15.3 \%$, and $100.0 \% \pm 9.4 \%$, respectively. This suggests that SPIOFC at the abovementioned concentration has no deleterious effects on the aforementioned cells.

Prussian blue staining for the presence of iron Both SPIOFC and SPIOF were located in the cytoplasm and became blue spots after Prussian blue staining. The cytoplasm of the U251-MG and C6 glioma cells cultured with SPIOFC (Figure 5B, 5D) contained lots of blue particles, whereas almost no blue spots were observed in the cytoplasm of corresponding glioma cells cultured with SPIOF (Figure 5A, 5C). The percentage of Prussian blue stained-positive (Figure 6A) U251-MG and C6 cells cultured with SPIOFC $(98.7 \% \pm 1.4 \%$ and $99.4 \% \pm 0.8 \%$, respectively) was significantly more than those of $251-\mathrm{MG}$ and $\mathrm{C} 6$ cells cultured with $\operatorname{SPIOF}(2.6 \% \pm 1.7 \%$ and $2.4 \% \pm 1.6 \%$, respectively). This result indicated U251-MG and $\mathrm{C} 6$ cells could specifically and efficiently internalize SPIOFC rather than SPIOF.

U251-MG and C6 cells cultured with SPIOFC (Figure 5B, 5D) took up a substantial amount of SPIOFC, whereas neural cells cultured with SPIOFC (Figure 5E) took up virtually no SPIOFC. The percentage of Prussian blue stained-positive (Figure 6B) U251-MG and C6 cells cultured with SPIOFC $(98.7 \% \pm 1.4 \%$ and $99.4 \% \pm 0.8 \%$, respectively) was significantly greater than those of neural cells cultured with SPIOFC $(0 \%)$. This result demonstrated that SPIOFC was specifically and efficiently taken up by U251-MG and C6 glioma cells rather than neural cells.

Quantification of nanoparticle intracellular uptake The ICP results were consistent with those obtained through Prussian blue staining. The iron uptake by U251-MG and C6 cells cultured with SPIOFC (72.5 \pm 1.8 and $74.9 \pm 2.2 \mathrm{pg} / \mathrm{cell}$, respectively) was much higher than those by $\mathrm{U} 251-\mathrm{MG}$ and

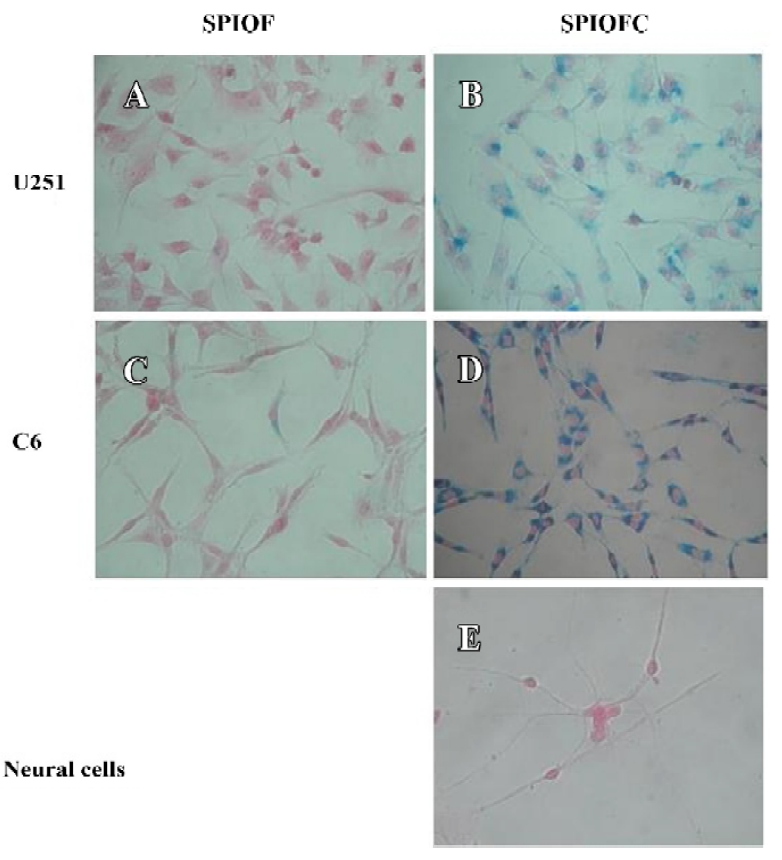

Figure 5. Prussian blue staining of U251-MG cells cultured with SPIOF (A) and SPIOFC (B); C6 cells cultured with SPIOF (C) and SPIOFC (D); neural cells cultured with SPIOFC (E). U251-MG and C6 cells took up lots of SPIOFC rather than SPIOF. More importantly, SPIOFC still could not be internalized by the neural cells $(\times 200)$.

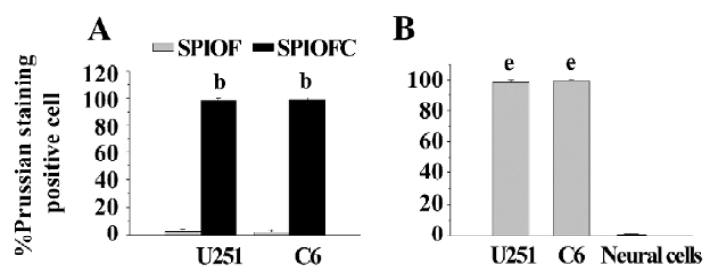

Figure 6. Percentage of Prussian blue stained-positive U251-MG and C6 cells cultured with SPIOF and SPIOFC (A). Percentage of Prussian blue stained-positive U251-MG, C6, and neural cells cultured with SPIOFC (B). $n=20$; Mean \pm SD. ${ }^{\mathrm{b}} P<0.05$ vs $\mathrm{SPIOF}$ group; ${ }^{\mathrm{e}} P<0.05$ $v s$ neural cells. 
C6 cells cultured with SPIOF $(6.6 \pm 1.0$ and $7.1 \pm 0.8 \mathrm{pg} / \mathrm{cell}$, respectively; Figure 7A). This result indicated that U251MG and C6 cells could specifically and efficiently internalize SPIOFC rather than SPIOF.

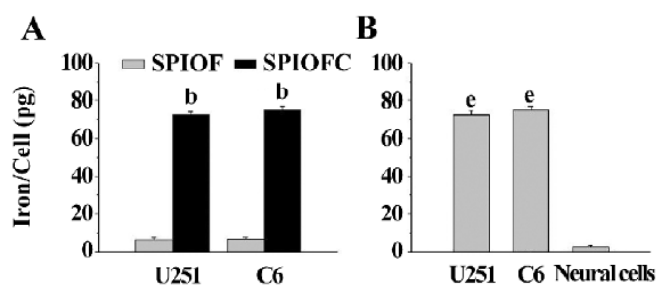

Figure 7. Iron uptake by $\mathrm{U} 251-\mathrm{MG}$ and $\mathrm{C} 6$ cells cultured with SPIOF and SPIOFC (A). Iron uptake by U251-MG, C6, and neural cells cultured with SPIOFC (B). $n=10$. Mean \pm SD. ${ }^{b} P<0.05$ vs SPIOF group; ${ }^{\mathrm{e}} P<0.05$ vs neural cells.

The iron uptake by U251-MG and C6 cells cultured with SPIOFC (72.5 \pm 1.8 and $74.9 \pm 2.2 \mathrm{pg} /$ cell, respectively) was much higher than those by neural cells cultured with SPIOFC ( $1.3 \pm 0.3 \mathrm{pg} /$ cell; Figure $7 \mathrm{~B})$. This result demonstrated that SPIOFC could specifically and efficiently be internalized by U251-MG and C6 glioma cells rather than neural cells.

MRI The MRI results were also consistent with those obtained through Prussian blue staining. The U251-MG and C6 cells cultured with SPIOFC (Figure 8B, 8D) showed a much greater negative contrast than the U251-MG and C6 cells cultured with SPIOF (Figure 8A, 8C). The $\mathrm{T}_{2}$ signal intensity (Figure 9A) of the U251-MG and C6 cells cultured with SPIOFC (233.6 \pm 25.9 and $211.4 \pm 17.2$, respectively) was substantially lower than those of the U251-MG and C6 cells cultured with SPIOF (2275.3 \pm 268.6 and $2342.7 \pm 222.4$, respectively). This result also demonstrated that U251-MG

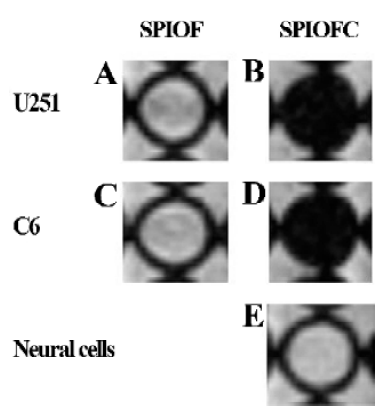

Figure 8. MRI of U251-MG cells cultured with SPIOF (A) and SPIOFC (B); C6 cells cultured with SPIOF (C) and SPIOFC (D); and neural cells cultured with SPIOFC (E). Cells were embedded in gelatin (1.5-T, $\mathrm{T}_{2}$ weighted spin-echo sequence, TR $3000 \mathrm{~ms}$, TE $20 \mathrm{~ms}$ ). U251-MG and $\mathrm{C} 6$ cells cultured with SPIOFC showed a much greater negative contrast than U251-MG and C6 cells cultured with SPIOF and neural cells cultured with SPIOFC. and C6 cells could specifically and efficiently internalize SPIOFC rather than SPIOF.

The U251-MG and C6 cells cultured with SPIOFC (Figure $8 \mathrm{~B}, 8 \mathrm{D})$ displayed a significantly greater negative contrast than neural cells cultured with SPIOFC (Figure 8E). The $\mathrm{T}_{2}$ signal intensity (Figure 9B) of the U251-MG and C6 cells cultured with SPIOFC (233.6 \pm 25.9 and $211.4 \pm 17.2$, respectively) was much lower than those of neural cells cultured with SPIOFC (2533.6 \pm 199.2$)$. This result also indicated that SPIOFC was specifically and efficiently taken up by U251MG and C6 cells rather than neural cells, and MRI in connection with SPIOFC had the potential to differentiate gliomas from normal brain tissue.

Confocal laser scanning microscopy SPIOFC internalized by U251-MG and C6 cells was observed as a green particle in confocal laser scanning microscopy (Figure 10). This suggests that SPIOFC has the ability to act as an optical imaging probe for visualizing gliomas.
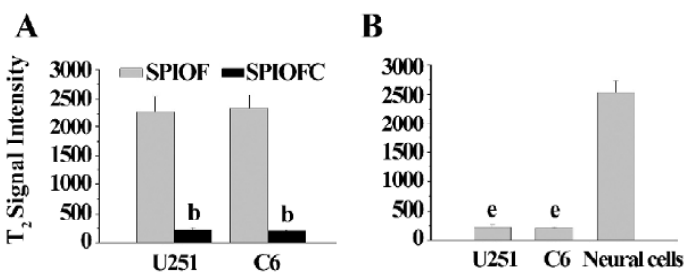

Figure 9. $\mathrm{T}_{2}$ signal intensity (A) of U251-MG and $\mathrm{C} 6$ cells cultured with SPIOF ( $n=13$ for U251-MG cells; $n=16$ for C6 cells) and SPIOFC $(n=17)$. MRI signal intensity (B) of U251-MG, C6, and neural cells $(n=10)$ cultured with SPIOFC. Mean \pm SD. ${ }^{\mathrm{b}} P<0.05$ vs SPIOF group; ${ }^{\mathrm{e}} P<$ 0.05 vs neural cells.
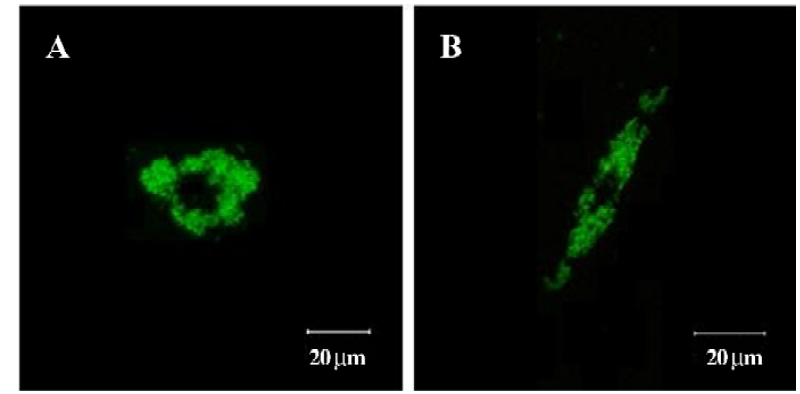

Figure 10. Confocal fluorescent images of U251-MG (A) and C6 cells (B) cultured with SPIOFC.

\section{Discussion}

The molecular imaging of SPIO-labeled glioma cells has the potential to achieve precise surgical resection of gliomas ${ }^{[3,7-10,22]}$ for the improved prognosis of patients. However, SPIO encountered low specificity for glioma cells and limited inter- 
nalization by glioma cells. To modify SPIO with chlorotoxin might be beneficial for enhanced specificity and efficiency of SPIO in labeling glioma cells. Moreover, the biocompatibility of modified SPIOFC is a prerequisite for biomedical application. The present study was therefore performed to determine: (i) whether chlorotoxin-conjugated SPIO (SPIOFC) is suitable for biomedical application; (ii) whether U251-MG and C6 glioma cells can be specifically and efficiently labeled by SPIOFC; and (iii) whether SPIOFC internalized by cells can be detected by both MRI and optical imaging. Our results demonstrated that SPIOFC, a safe contrast agent, was a specific and efficient targeting contrast agent for gliomas, and MR and optical imaging could effectively detect SPIOFClabeled glioma cells.

Theoretically, excessive iron maybe harmful to cell survival. However, what is considered the safe application range for SPIO concentrations remains unclear. Our study investigated the effect of SPIO at concentration of 5, 10, 25, 50, 75, 100 , and $200 \mathrm{mg} / \mathrm{L}$ on cell viability. The results demonstrated that SPIO at the abovementioned concentrations did not impair cell viability. Previous studies demonstrated that chlorotoxin can inhibit the growth of gliomas ${ }^{[17,18]}$. In this study, we found that chlorotoxin at low concentrations $(0.8$ and $1.6 \mu \mathrm{mol} / \mathrm{L}$ ) did not impair the viability of glioma cells, whereas chlorotoxin at higher concentrations $(2.4,3.2,4.0,4.8$, and $5.6 \mu \mathrm{mol} / \mathrm{L}$ ) had cytotoxic effects on glioma cells. These results were consistent with Deshane et al's report ${ }^{[18]}$. Chlorotoxin at all the abovementioned concentrations did not impair the viability of neural cells. This might due to the fact that there was no chlorotoxin specific receptor (MMP-2) on the surface of the neural cells. Therefore, SPIO and chlorotoxin, utilized for the synthesis of SPIOFC, was controlled at concentrations of $100 \mathrm{mg} / \mathrm{L}$ (iron concentrations) and $1.6 \mu \mathrm{mol} / \mathrm{L}$, respectively. The MTT results showed that SPIOFC at this concentration was safe for cell survival. This might be due to the fact that SPIO $(100 \mathrm{mg} / \mathrm{L}$ iron concentrations) and chlorotoxin $(1.6 \mu \mathrm{mol} / \mathrm{L}) \mathrm{did}$ not impair the viability of glioma and neural cells. The viability of the neural cells cultured with SPIOFC (100 mg/L) was higher than the corresponding control groups, which is consistent with another report ${ }^{[28]}$. The mechanism for this phenomenon needs to be further investigated.

In this study, U251-MG and C6 glioma cells took up lots of SPIOFC, whereas SPIOF had difficulty entering the cytoplasm of both cells by internalization. Moreover, in comparison with glioma cells, neural cells virtually did not contain SPIOFC despite being cocultured with SPIOFC for an adequate duration. This demonstrates that SPIOFC has high specificity and efficiency in labeling glioma cells. Chlorotoxin, a small peptide, should be responsible for the marked advantage and prospect of SPIOFC in the cell-level differentiation of gliomas from neural cells. MMP-2, a specific receptor of chlorotoxin, only exists on the membrane of glioma cells rather than normal brain tissue cells, including neural cells. In glioma cells, the close connection between MMP-2 and chlorotoxin contributes to the transplantation of SPIOFC into intracellular space across the cellular membrane via receptor-mediated endocytosis. According to our estimate, it is hard for SPIOF to be linked to the surface of both glioma and neural cells. More importantly, the MR and optical imaging of glioma cells were distinct from those of neural cells after SPIOFC was used to treat 2 cell types. Thus, MRI and optical imaging might be a feasible and effective tool for discriminating gliomas from surrounding normal brain tissue in cell level during preoperative and intraoperative procedures, respectively.

The preferential uptake of chlorotoxin nanoparticles conjugated by 9L gliosarcoma cells has been reported using cardiomyocytes as control cells ${ }^{[22]}$, and our data are consistent with those observations. However, the present study investigated the influence of SPIOFC on glioma cells originated from humans and rats. The species did not affect the efficacy of SPIOFC in the specific labeling of glioma cells. The U251-MG cell line is glioma cell line of human origin. These provide the insight into the future clinical application of SPIOFC. The biocompatibility of SPIOFC was assessed in this study, and the encouraging results indicated that SPIOFC was suitable for biomedical applications. Moreover, the present study utilized neural cells as the control for glioma cells, which imitated the histological difference between central tumors and peripheral normal brain tissue in the setting of clinical practice. Previous studies employed a 4.7-T or 3.0-T MR imager to assess the ability of SPIO in visualizing gliomas $^{[3,10,22]}$. In our study, a clinical 1.5-T MR imager was selected. As a result, the 1.5-T MR scanner in conjunction with SPIOFC was sufficient to produce a visual contrast between gliomas and normal brain tissue. These facilitate the wide application of SPIOFC in clinical practice. Nevertheless, the in vivo biodistribution, metabolic dynamics, and targeting effects of SPIOFC remain to be clarified. Those issues are currently under investigation in our laboratory.

In conclusion, both SPIOFC and SPIO are safe contrast agents for glioma and neural cells. However, SPIOFC is more suitable for the specific and efficient targeting of glioma cells than SPIO, because chlorotoxin play a critical role in the specific and efficient transporting of functional SPIO into glioma cells rather than neural cells. As a result, MRI and optical imaging in conjunction with SPIOFC in vitro can 
differentiate glioma cells from normal brain tissue cells.

\section{Acknowledgements}

We are grateful to professor Keiji KAWAMOTO (Department of Neurosurgery, Kansai Medical University, Osaka, Japan) for providing the glioma cell lines.

\section{References}

1 Wrensch M, Minn Y, Chew T, Bondy M, Berger MS. Epidemiology of primary brain tumors: current concepts and review of the literature. Neuro Oncol 2002; 4: 278-99.

2 Meng QH, Zhou LX, Luo JL, Cao JP, Tong J, Fan SJ. Effect of 7hydroxystaurosporine on glioblastoma cell invasion and migration. Acta Pharmacol Sin 2005; 264: 492-9.

3 Muldoon LL, Sandor M, Pinkston KE, Neuwelt EA. Imaging, distribution, and toxicity of superparamagnetic iron oxide magnetic resonance nanoparticles in the rat brain and intracerebral tumor. Neurosurgery 2005; 57: 785-96.

4 Jung CW, Jacobs P. Physical and chemical properties of superparamagnetic iron-oxide MR contrast agents: ferumoxides, ferumoxtran, ferumoxsil. Magn Reson Imaging 1995; 13: 661-74.

5 Chouly C, Pouliquen D, Lucet I, Jeune JJ, Jallet P. Development of superparamagnetic nanoparticles for MRI: effect of particle size, charge and surface nature on biodistribution. J Microencapsul 1996; 13: 245-55.

6 Jing M, Liu XQ, Liang P, Li CY, Zhang XT, Wang D, et al. Labeling neural stem cells with superparamagnetic iron oxide in vitro and tracking after implantation with MRI in vivo. Zhonghua Yi Xue Za Zhi 2004; 84: 1386-9 (Chinese).

7 Fleige G, Nolte C, Synowitz M, Seeberger F, Kettenmann H, Zimmer C. Magnetic labeling of activated microglia in experimental gliomas. Neoplasia 2001; 3: 489-99.

8 Knauth M, Egelhof T, Roth SU, Wirtz CR, Sartor K. Monocrystalline iron oxide nanoparticles: possible solution to the problem of surgically induced intracranial contrast enhancement in intraoperative MR imaging. AJNR Am J Neuroradiol 2001; 22: 99102.

9 Moore A, Marecos E, Bogdanov A Jr, Weissleder R. Tumoral distribution of long-circulating dextran-coated iron oxide nanoparticles in a rodent model. Radiology 2000; 214: 568-74.

10 Kircher MF, Mahmood U, King RS, Weissleder R, Josephson L. A multimodal nanoparticle for preoperative magnetic resonance imaging and intraoperative optical brain tumor delineation. Cancer Res 2003; 63: 8122-5.

11 Hunt MA, Bago AG, Neuwelt EA. Single-dose contrast agent for intraoperative MR imaging of intrinsic brain tumors by using ferumoxtran-10. AJNR Am J Neuroradiol 2005; 26: 1084-8.

12 Moore A, Basilion JP, Chiocca EA, Weissleder R. Measuring transferrin receptor gene expression by NMR imaging. Biochim Biophys Acta 1998; 1402: 239-49.
13 Lowry MB, Duchemin AM, Robinson JM, Anderson CL. Functional separation of pseudopod extension and particle internalization during Fc gamma receptor-mediated phagocytosis. J Exp Med 1998; 187: 161-76.

14 Zhang Y, Kohler N, Zhang MQ. Surface modification of superparamagnetic magnetite nanoparticles and their intracellular uptake. Biomaterials 2002; 23: 1553-61.

15 Peng JF, Wang KM, Tan WH, He XX, He CM, Wu P, et al. Identification of live liver cancer cells in a mixed cell system using galactose-conjugated fluorescent nanoparticles. Talanta 2007; 71: 833-840.

16 DeBin JA, Strichartz GR. Chloride channel inhibition by the venom of the scorpion Leiurus quinquestriatus. Toxicon 1991; 29: 1403-8.

17 Lyons SA, O’Neal J, Sontheimer H. Chlorotoxin, a scorpionderived peptide, specifically binds to gliomas and tumors of neuroectodermal origin. GLIA 2002; 39: 162-73.

18 Deshane J, Garner CC, Sontheimer H. Chlorotoxin inhibits glioma cell invasion via matrix metalloproteinase-2. J Biol Chem 2003; 278: 4135-44.

19 Kachra Z, Beaulieu E, Delbecchi L, Mousseau N, Berthelet F, Moumdjian R, et al. Expression of matrix metalloproteinases and their inhibitors in human brain tumors. Clin Exp Metastasis 1999; 17: 555-66.

20 Soroceanu L, Gillespie Y, Khazaeli MB, Sontheimer H. Use of chlorotoxin for targeting of primary brain tumors. Cancer Res 1998; 58: 4871-9.

21 Shen S, Khazaeli MB, Gillespie GY, Alvarez VL. Radiation dosimetry of ${ }^{131} \mathrm{I}$-chlorotoxin for targeted radiotherapy in gliomabearing mice. J Neurooncol 2005; 71: 113-9.

22 Veiseh O, Sun C, Gunn J, Kohler N, Gabikian P, Lee D, et al. Optical and MRI multifunctional nanoprobe for targeting gliomas. Nano Lett 2005; 5: 1003-8.

23 Cai W, Wan JQ. Facile synthesis of superparamagnetic magnetite nanoparticles in liquid polyols. J Colloid Interface Sci 2007; 305: 366-70.

24 Wan JQ, Cai W, Feng JT, Meng XX, Liu EZ. In situ decoration of carbon nanotubes with nearly monodisperse magnetite nanoparticles in liquid polyols. J Mater Chem 2007; 17: 1188-92.

$25 \mathrm{Lu} \mathrm{Y,} \mathrm{Yin} \mathrm{YD,} \mathrm{Mayers} \mathrm{BT,} \mathrm{Xia} \mathrm{YN.} \mathrm{Modifying} \mathrm{the} \mathrm{surface} \mathrm{pro-}$ perties of superparamagnetic iron oxide nanoparticles through a sol-gel approach. Nano Lett 2002; 2: 183-6.

26 Sun EY, Josephson L, Kelly KA, Weissleder R. Development of nanoparticle libraries for biosensing. Bioconjug Chem 2006; 17 : 109-13.

$27 \mathrm{Yu} \mathrm{X,} \mathrm{An} \mathrm{L.} \mathrm{A} \mathrm{serum-} \mathrm{and} \mathrm{antioxidant-free} \mathrm{primary} \mathrm{culture} \mathrm{model}$ of mouse cortical neural cells for pharmacological screen and studies of neurotrophic and neuroprotective agents. Cell Mol Neurobiol 2002; 22: 197-206.

28 Cheng FY, Su CH, Yang YS, Yeh CS, Tsai CY, Wu CL, et al. Characterization of aqueous dispersions of $\mathrm{Fe}_{3} \mathrm{O}_{4}$ nanoparticles and their biomedical applications. Biomaterials 2005; 2: 72938 . 